\title{
The Person-Centered Health model in Intellectual Developmental Disorders/Intellectual Disability
}

\author{
Marco O. Bertellia,* \\ Annamaria Bianco ${ }^{a}$ \\ Micaela Piva Merlia \\ Luis Salvador-Carullab,c \\ a CREA (Research and Clinical Centre), \\ Fondazione San Sebastiano, \\ Florence \\ ${ }^{b}$ Centre for Disability Research and Policy, \\ Faculty of Health Sciences, University \\ of Sydney \\ ${ }^{c}$ Mental Health Policy Unit, Brain and Mind \\ Institute, Faculty of Health Sciences, \\ University of Sydney
}

ITALY

AUSTRALIA

\begin{abstract}
Background and Objectives: This paper analyses the different aspects related to the conceptualization and assessment of Intellectual Developmental Disorders / Intellectual Disability (IDD/ID) following the Person-centered Integrative Diagnostic (PID) model of the International Network for Person-centered Medicine, with a main emphasis on the health status and health self-perception.

Methods: Conceptual paper, including expert opinions based on literature review.

Results: The conceptualization of IDD/ID should shift the traditional over-reliance on the intelligence (IQ) score in favour of the daily life expression of specific cognitive functions and the determination of the levels of severity of intellectual functioning, that is currently based on the person's IQ score, should be reached through a system that is predicated on the person's satisfaction attainment towards life. The assessment of cognition should be aimed at identifying those dysfunctions that have the highest impact on individual behaviour, skills, adaptation, autonomy, and quality of life across the life span, highlighting personal cognitive strengths and weaknesses that can be worthwhile for the planning of effective interventions.

Conclusions: Authors conclude that the application of the PID model to IDD/ID represents a prototypical example of how this approach can be useful for understanding complex constructs in health care. An overview of the main factors related to the implementation of the person-centered care model by health systems and services is also provided.
\end{abstract}




\section{Intellectual developmental Disorders and the Person-centered Integrative Diagnostic model}

In 2010 the International Network for Person-centered Medicine proposed a Personcentered Integrative Diagnostic model (PID) defined by broad informational domains, covering both ill health and positive health along three levels: health status (disease/ disability versus wellbeing); experience of health (aspirations, satisfaction, experiences of ill-being and of wellbeing); and contributors to health (risk and protective factors) ${ }^{1}$. According to this model, diagnosis is both a process and a formulation, and it should be oriented by the articulation of science and humanism, the use of a bio-psycho-socialcultural-spiritual framework.

This conceptual framework developed in mental health field has been refined during the last five years in the context of the Geneva Conferences and Declarations produced by the International College of Person-Centered Medicine (ICPCM). However, its ap- plicability to Intellectual Developmental Disorders or Intellectual Disabilities (IDD/ID) remains unexplored. This is particularly relevant due to the complexity of this condition, and the challenges described in its assessment, diagnosis and care planning; in spite of the fact that the IDD/ID sector was an earlier adopter of the person-centered care model ${ }^{2-4}$.

The different domains of the PID model of person-centered health show particular challenges in IDD/ID. The contributors or determinants of health in IDD/ID have been extensively analysed, including factors such as deprivation, ethnicity and health inequalities $^{5,6}$.

In this paper we analyse the different aspects related to the conceptualisation and assessment of IDD/ID following the PID approach to person-centered health with a main emphasis on the health status and health self-perception of persons with IDD/ID. In the last section of this paper we provide an overview of the main factors related to the implementation of the personcentered care model by care and support organisations in this field.

Table 1

Domains, sub-domains and items content of the QOL-IP to persons with IDD (Italian version Bertelli et al. ${ }^{46}$ ).

\begin{tabular}{|c|c|c|}
\hline Domains & Sub-domains & Content of items \\
\hline \multirow{3}{*}{ Being } & Physical & Appearance, grooming, hygiene, nutrition, fitness, mobility, sleep, sex \\
\hline & Psychological & $\begin{array}{l}\text { Psychological health and adjustment, feelings, self-concept, self-esteem, } \\
\text { self-consciousness }\end{array}$ \\
\hline & Spiritual & Spiritual beliefs, personal values, standards of conduct, hope for the future \\
\hline \multirow{3}{*}{ Belonging } & Physical & $\begin{array}{l}\text { Relationship with physical environments: home, neighbourhood, } \\
\text { community, work }\end{array}$ \\
\hline & Social & $\begin{array}{l}\text { Relationship with others: partner/spouse, friends, family, co-workers, } \\
\text { neighbours, groups }\end{array}$ \\
\hline & Community & $\begin{array}{l}\text { Access to public events and resources (work, income, services/programs, } \\
\text { community events) }\end{array}$ \\
\hline \multirow{3}{*}{ Becoming } & Practical & $\begin{array}{l}\text { Daily/regular activities: domestic, voluntary, work, school, attending } \\
\text { appointments (health) }\end{array}$ \\
\hline & Leisure & $\begin{array}{l}\text { Leisure and recreational activities: hobbies, socializing, vacations, holiday } \\
\text { activities }\end{array}$ \\
\hline & Growth & $\begin{array}{l}\text { Improving/learning skills, adapting to life's changes, trying new things, } \\
\text { problem-solving }\end{array}$ \\
\hline
\end{tabular}




\section{Health Status of persons with Intellectual Developmental Disorders}

The PID model highlights the importance of the assessment of the positive domain of wellbeing and good functioning, as well as the negative domain which includes the health condition or disease and the status of disability. The relationship between disability and functioning in the person-centered medicine model has been revised elsewhere ${ }^{7}$.

The case of IDD/ID is particularly challenging as there is not an international consensus on whether it is a health condition and therefore it should be classified in the International Classification of Diseases (ICD) or it is a disability that should not be coded in ICD but in the International Classification of Functioning (ICF). The fact that the international community has not reached an agreement in such a basic question and that there is an ongoing debate on its placement at the WHO Family of Classifications reflects the complexity of this construct. As a matter of fact many national agencies follow the approach of the American Association of Intellectual and Developmental Disabilities that defines "Intellectual disability" as "a disability characterized by significant limitations both in intellectual functioning [IQ>70] and in adaptive behaviour, which covers many everyday social and practical skills. The timeframe for age of onset is defined from birth to 18 years. This has oriented the recommendations made by this group for naming and conceptualising this condition at $\mathrm{ICD}^{8,9}$. Other organisations such as the World Psychiatric Association have defined it as a group of health condition, namely developmental conditions, characterized by a significant impairment of cognitive functions, which are associated with limitations of learning, adaptive behaviour and skills ${ }^{10}$. This definition was extended by the
ICD Working Group in 2011, under the new term of "Intellectual Developmental Disorders": a group of etiologically diverse conditions that are present from birth or that arise during the developmental period, characterized by a marked impairment of cognitive functions necessary for the development of knowledge, reasoning, and symbolic representation of the level expected of one's age peers, cultural and community environment. IDD was also defined as a life span condition requiring consideration of all developmental stages and life transitions ${ }^{11,12}$. Furthermore, the ICD Working Group proposed revised diagnostic criteria for ICD-11 based on an articulated model of cognitive impairment. This approach should complement measurement of IQ with assessment of specific cognitive functions and a contextualized description of consequent adaptive and learning difficulties. Within this new approach, cognitive skills should be assessed in the most comprehensive way as possible. The measurement should be done through tests, semistructured observations, and direct clinical examination. The tests should combine measurement of IQ with that of complex aspects of executive functioning, including perceptual reasoning, processing speed, verbal comprehension, as well as specific aspects, such as attention, perception, and working memory. The evaluation should aim to identify the cognitive dysfunctions that have the greatest negative impact on the person's lifespan, not only in cognitive skills but also in terms of associated behaviour, ability, adjustment, autonomy, and all other factors that rely on person-centered health ${ }^{11,12}$.

The current debate reflects the importance of a multifaceted approach complex health problems and the significant contribution made by ICPCM and the PID integrative multidimensional matrix ${ }^{1}$, which takes into account not only the health condition and the disability but also the positive aspects de- 
fined as wellbeing and good functioning. IDD/ID may not be an exception in health but a prototypical example of how the holistic and comprehensive approach recommended by ICPCM is useful for understand these complex constructs in health care.

\section{Analysis of self-reported wellbeing and satisfaction in IDD}

Quality of Life (QoL) is a term that has become widely used in everyday language. It is understood at a general level to refer to the "good things" of life, and, when applied to individuals, to high attainment and satisfaction in specific areas of life that are of particular interest or concern to individuals at their current stage of life. The public media, especially, promotes a simpler view, typically referring quite clearly to high level of life quality or the "best" of life. This popularization of the term QoL has resulted in it being understood and used in ways that are considerably less substantial than the conceptualizations that have been described and applied by academics and researchers. For this reason, it is sometimes considered that QoL is a concept that lacks sufficient precision to have specific usefulness in the field of IDD/ID, and that it is more advantageous to focus on clearer concepts or simply on providing practical supports ${ }^{13}$.

Yet, a considerable amount of substantive work has been done in the area of QoL and IDD/ID. Numerous thoughtful books, book chapters, and academic articles have been published that not only advanced our understanding of QoL, but have made ID the leading field for substantive work in QoL. It is clear from this work that achieving high quality of living is not the main purpose.
Rather, it provides an approach for how professionals view the patient-person, and for the relationship between the patient-person and the system of care. The QoL approach, then, consists more of chosing a course than in achieving a goal.

The QoL approach provides both a viable and an alternative approach to the traditional medical approach. The QoL approach tries, overall, to help people be satisfied with their own lives in ways that are customary to them and valued by them, while the latter focuses primarily on restoring morphological and functional integrity to aspects of the person that have been affected by the morbid condition. The QoL approach begins by professionals understanding what is important to and valued by each individual, and what aspects of life or the environment contribute positively to life quality or detract from it. This approach makes every effort to respect the right of the individual to help choose the course of action that best suits him or her, and endeavours to provide whatever positive supports are required to assist the individual to live an effective life that is uniquely shaped by individual characteristics and circumstances ${ }^{14}$. One of the fundamental principles of the QoL approach is that QoL is important for all people, and that all people are thus entitled to a life of quality. This principle applies equally to people with IDD/ID and to people without this condition.

QoL has been defined by the WHO as the “individuals' perception of their position in life in the context of the culture and value systems in which they live and in relation to their goals, expectations, standards and concerns. It is a broad ranging concept, incorporating in a complex way individuals' physical health, psychological state, level of independence, social relationships, personal beliefs and their relationships to salient features of the environment"15. 
QoL measurement often relies on people's own perceptions and expressions of their well-being or general satisfaction with life, referred to in the measurement literature as Subjective Well-Being (SWB). It has two components, the individual's expressions of positive or negative emotions or mood (happiness) and the individual's general satisfaction with several aspects of his/her life (satisfaction $)^{16}$. Supportive family and friends, work, satisfaction with the self, religious or spiritual life, learning and growth, leisure and health are components that go into most people's experience of satisfaction ${ }^{17,18}$.

Since life is experienced by individuals through their unique sets of perceptions, SWB may be an essential pathway to recording "true" personal QoL. However, when recording SWB, it is often noted that QoL scores of people with severe disorders or disabilities do not significantly differ from those of the general population. This seeming paradox has been explained in various ways ${ }^{19}$, such as methodological bias, or the psychological mechanism of adaptation, coping or resilience. Cummins has presented a compelling case for SWB as the result of an affective/cognitive homeostatic system that is characteristic of humans. Simply put, we have a built-in tendency to assess the world around us, and our place within that world, in normative ways by perceiving that "life is okay." Only in extreme cases, where there is an strong overload, does the homeostatic system fail. Thus, SWB may not be a particularly sensitive measure, but, when it is low, it may be cause for serious concern ${ }^{20}$.

The WHO definition of QoL as a subjective construct including the self-reported assessment of both positive and negative facets of life is in agreement with the "experience" domain of the PID model of personcentered health ${ }^{1}$. However the PID has a broader perspective as it highlights the im- portance of engagement, empathy and partnership in the clinical care process, and sustain the patients' autonomy, responsibility and dignity while advancing the recovery and promotion of wellbeing. To assess the domain of person's experience and values PID uses descriptive categories, dimensions, and narratives, to cultivate patient-familyclinician partnerships for achieving shared diagnostic understanding and shared commitment to care. The application of this model to the assessment of personal wellbeing, experiences, satisfaction and aspirations of persons with IDD faces significant challenges in persons with IDD as the selfreported assessment of these complex concepts is limited due to the cognitive and communication impairments ${ }^{12}$.

It is important to take into account that there are other approaches to QoL in IDD/ID. Robert Schalock and colleagues developed a "Quality of Life Index" for intellectual disabilities within the context of the positive approach to this ${ }^{21}$ and the American Association of Intellectual and Developmental Disabilities (AAIDD), former American Association of Mental Retardation (AAMR) formalised the AAMR/AAIDD multidimensional model of human functioning. However Shalock's underlying social concept of QoL is very different from other approaches to health-related QoL ${ }^{13,22}$. Shalock defines QoL as "a multidimensional construct that has both subjective and objective components and is influenced by personal and environmental factors" 23 .

\section{Self-reported experiences and satisfaction in persons with Mild/Moderate IDD}

The use of self-reported assessment of QoL domains has been mainly documented in persons with mild-to-moderate intellectual disability (ID). A study analysing five in- 
dicators of subjective well-being and the personal characteristics, socio-economic position, and social connectedness found only slightly lower subjective well-being than typically reported among the general population. Variation in subjective well-being was strongly and consistently related to socio economic disadvantage and social relationships appraised self-reported well-being and satisfaction in persons with IDD and found benefits of residential support provided in very small settings, with choice of where and with whom to live and to individuals living with family ${ }^{24,25}$.

The role of satisfaction in QoL of IDD has been emphasised by several authors ${ }^{26}$. Lucas and Salvador-Carulla ${ }^{18}$ have shown the usability of self-reported measures of satisfaction in mild-moderate ID using the Satisfaction with Life Scale (SWLS) ${ }^{27}$. Persons with IDD were satisfied with their life, and associations were confirmed between SWLS and the Overall QoL, satisfaction with health and WHOQOL-BREF. SWLS scores significantly discriminated between healthy and unhealthy; and those reporting higher satisfaction with their relationships, home environment and their jobs compared to participants with lower satisfaction levels ${ }^{18}$.

\section{Self-reported experiences and satisfaction in persons with Severe IDD}

Even though there is an agreement on the applicability of the main domains of QoL to anybody's life, including severe IDD $^{28}$, the measurement of QoL in people with severe IDD is more complex due to the extent of cognitive and communication impairments that difficult direct interviews and self-assessments ${ }^{29}$. A number of studies indicate that consistent behavioural repertoires can be interpreted by proxies and validated by more independent others as expression of preference and satisfaction ${ }^{30}$.

Recently a four rounds Delphi process including 12 experts was conducted to develop a set of QoL indicators appropriate to adults with severe disabilities, using and the eight-domain conceptual model proposed by Schalock \& Verdugo. Each proposed item was evaluated according to the criteria of suitability, importance, observability and sensitivity. A final pool of 118 items was produced with the experts' strongest agreement for the items related to material wellbeing, while the weakest was found for items related to personal development ${ }^{31}$.

The usability of generic QoL measures may need further research from both qualitative and quantitative aspects. Some authors showed low correlations between QoL scores obtained through direct interview and the ones from proxies ${ }^{32,33}$ while others found good correspondence ${ }^{34-37}$. The impact that the presence of severe ID may have on front-line support workers in QoL rating hasn't been expressly studied yet.

Bertelli and collaborators ${ }^{38}$ have explored the use of a generic QoL rating scale (Quality of Life Instrument Package - QoL-IP ${ }^{39}$ ) in people with severe ID and normal healthy subjects. They found scores of life satisfaction in persons with ID higher than expected and similar to those of the general population. This data could be explained in terms of low expectations, little choice, lack of experience $^{40}$, and, as previously mentioned, low sensitivity of self wellbeing assessments, the latter due to an inner affective/cognitive homeostatic system, which would tend to be maintained at normative levels ${ }^{20}$. "Being Psychological" came out to be very low in the group with ID: this is an important fact, because this aspect is not always properly estimated. Concerning the moderating influ- 
ences, the group with IDD/ID shows more opportunities except for "Belonging Spiritual" and "Belonging Social". That could be in relation with the former data concerning the "Being Psychological". It's important to remind that "Decision-making" of people with ID is inferior in every domains compared to the control group ${ }^{38}$.

Taking a holistic approach to QoL, objective conditions and personal satisfaction appear to be distinct ${ }^{41}$. On the other hand some data show a positive correlation between selfdetermined behaviours and QoL scores so strong to let suppose for the former a predictive variable value for high QoL scores ${ }^{38,42}$.

\section{A theoretical model to ensure entitlement to a life of quality for people with ID}

The question of how we can ensure entitlement to a life of quality for people with ID who have mental health problems is an interesting and challenging one. Yet, the emphasis within the conceptualization of QoL on finding uniquely individual solutions to presenting problems suggests that it may be a particularly apt approach to take with this population. People with ID are characterized by lower levels of some cognitive functions, and lower potential for learning and developing logical-deductive skills. For this reason, it is not a sane goal of therapeutical interventions to attempt to provide "normal" intellectual functioning, but rather such interventions need to work within the confines of each individual's particular skills sets. Similarly, it is not a reasonable therapeutic goal to have people with IDD/ID live their lives as close as possible to people without IDD/ID, because ultimately they have to find satisfaction in those aspects of life that are important to them and that they can understand and experience. The more traditional medical ap- proach to therapeutic interventions with people who have IDD/ID and mental health problems have tended not to consider the uniqueness of people with IDD/ID fully and have been more likely to use the general population norm as their point of reference. This has resulted in interventions that have dealt primarily with containing troublesome behaviours and helping to alleviate symptoms, rather than to address the root causes of the problems. Thus, assuming an overall QoL approach to intervention goes a long way to ensuring entitlement to a life of quality.

The conceptualisation of ID should shift the traditional over-reliance on the intelligence (IQ) score in favour of the daily life expression of specific cognitive functions and the determination of the levels of severity of intellectual functioning, that was previously based on the person's IQ score, should be reached through a system that is predicated on the person's satisfaction attainment towards life.

The assessment of cognition should be aimed at identifying those dysfunctions that have the highest impact on individual behaviour, skills, adaptation, autonomy, and quality of life across the life span, highlighting personal cognitive strengths and weaknesses that can be useful to understand personal functioning and to organize intervention.

\section{The Person-centered Care model in the provision of care and support for IDD}

Person-centeredness as a model of care provision, care individualisation and lifestyle and supports was first developed in the ID sector than in many other areas of health and social care. However, and although some agencies have actually implemented 
PCC over decades in the US, Australia and Europe, the claims of adherence to the PCC goals are larger than its actual application $^{43,44}$.

As a matter of fact and in spite of the aspirations towards a PCC model, the implementation is slow due to barriers in the change in the philosophy or culture of care, power and funding structures, high levels of staff turnover and lack of training, inexperience among service management, inadequate staff supervision, and ambiguity among some stakeholders ${ }^{45}$.

Kendrick $^{44}$ has provided an extensive review of the main 7 conditions required for such a change in the service delivery system in IDD care (Table 2). The challenges related to this change include 1) Ethical Partnering With Individuals and Families and Carers; 2) A Social Inclusion /Valued Social Participation; and 3) Effective Address of People's Important Personal Needs.

Building partnerships that work requires a staff that understands ethical conduct and its aware of its absence or perversion, incorporates decision sharing, personal and organizational flexibility and demonstrates respect for persons with ID. The social inclusion/ participation approach requires moving out from segregated services to fully community integration and membership, providing support for the acquisition of common social and cultural values as well as health promotion through healthy lifestyles and prevention of stigma and marginalisation. The effective address of personal needs requires a monitoring system of care and support gaps, its relation to unmet needs, with an specific attention to "the essential ingredients of a good life such as employment, income, good health, a home of their own, value and respect, interesting leisure, personal growth and development, good safeguarding of vulnerabilities" 44 .

To achieve these goals it is necessary to invest in the assistance to establish service user controlled entities to ensure consumer empowerment; independent personal advocacy; oversight and evaluation of services and service quality agreements directed by service users in terms of agency performance; and provide funding on the personcentered approach in education, leadership and to increase population literacy in PCC.

Table 2

Organisation capabilities required for the Implementation of a Person centered Care model in Intellectual Disabilities (adapted from Kendrick ${ }^{44}$ ).

1. Capacity to routinely create individualized options with a person "from scratch".

2. Convert existing group or fixed models of service to individualized ones as requested.

3. Capacity for creating these options (innovation), sustainability and change.

4. Generate an individualized option for all persons being served, including persons who might be considered notably poor candidates for individualization i.e. persons who are allegedly "difficult to serve.

5. Sustain these individualized options across multiple jurisdictions and time periods with varying political, economic and leadership climates (i.e. under all likely conditions- robustness).

6. Capacity to deliver individualized arrangements at an average cost that would be competitive in the aggregate with aggregate costs for group or fixed models of service.

7. Assure comparability and safety of individualized arrangements created for the people being supported relative to their specific vulnerabilities via the use intentional safeguards. 


\section{Ackowledgments}

This study follows the contribution of $\mathrm{MB}$ and LSC to the TRINNODD project: LU/08/LLP/LdV-TOI-156002 within the Leonardo da Vinci programme of the European Commission.

\section{References}

1. Mezzich JE, Salloum IM, Cloninger CR, SalvadorCarulla L, Kirmayer LJ, Banzato CE, et al. Person-centred integrative diagnosis: conceptual bases and structural model. Can J Psychiatry. 2010; 55(11):701-8.

2. Callan L, Gilbert T, Golding K, Lockyer T, Rafter K. Assessing health needs in people with severe learning disabilities: a qualitative approach. J Clin Nurs. 1995; 4(5): 295-302.

3. Turner S, Cash J. The effectiveness of services for people with learning disabilities. Nurs Times. 1999; 95(26): 48-9.

4. Holburn S, Vietze P. Acknowledging barriers in adopting person-centered planning. Ment Retard. 1999; 37(2): 117-24.

5. Emerson E. Deprivation, ethnicity and the prevalence of intellectual and developmental disabilities. J Epidemiol Community Health. 2012; 66(3): 218-24.

6. Emerson E. The Rio Declaration: addressing the health inequalities experienced by people with intellectual disabilities. J Intellect Disabil Res. 2012; 16(2): 83-4.

7. Salvador-Carulla L, Gasca VI. Defining disability, functioning, autonomy and dependency in person-centered medicine and integrated care. Int J Integr Care. 2010; 10 Suppl:e025.

8. Tassé MJ, Luckasson R, Nygren M. AAIDD proposed recommendations for ICD-11 and the condition previously known as mental retardation. Intellect Dev Disabil. 2013; 51(2): 127-31.

9. Wehmeyer ML, Buntinx WH, Lachapelle Y, Luckasson RA, Schalock RL, Verdugo MA., et al. The intellectual disability construct and its relation to human functioning. Intellect Dev Disabil. 2008; 46(4): 311-8.

10. Salvador-Carulla L, Bertelli M. 'Mental retardation' or 'intellectual disability': time for a conceptual change. Psychopathology. 2008; 41(1): 10-6.
11. Salvador-Carulla L, Reed GM, Vaez-Azizi LM, Cooper SA, Martinez-Leal R,Bertelli M et al. Intellectual developmental disorders: towards a new name, definition and framework for "mental retardation/intellectual disability" in ICD-11. World Psychiatry. 2011; 10(3): 175-80.

12. Bertelli MO, Salvador-Carulla L, Scuticchio D, Varrucciu N, Martinez-Leal R, Cooper SA et al. Moving beyond intelligence in the revision of ICD-10: specific cognitive functions in intellectual developmental disorders. World Psychiatry. 2014; 13(1): 93-4.

13. Bertelli M, Brown I. Quality of life for people with intellectual disabilities. Curr Opin Psychiatry. 2006; 19(5): 508-13.

14. Brown I. Brown RI. Quality of life and disability: an approach for community practitioners. London: Jessica Kingsley Publishers. 2003.

15. The World Health Organization Quality of Life assessment (WHOQOL): position paper from the World Health Organization. Soc Sci Med. 1995; 41(10): 1403-9.

16. Diener E, Suh EM. Measuring quality of life: economic, social, and subjective indicators. Soc Indic Res. 1997; 40: 189-216.

17. Diener E, Suh EM, Lucas RE, Smith HL. Subjective Well-Being: Three Decades of Progress. Psychol Bull. 1999; 125: 276-302.

18. Lucas-Carrasco R, Salvador-Carulla L. Life satisfaction in persons with intellectual disabilities. Res Dev Disabil. 2012; 33(4): 1103-9.

19. Albrecht GL, Devlieger PJ. The disability paradox: high quality of life against all odds. Soc Sci Med. 1999; 48(8): 977-88.

20. Cummins RA. Moving from the quality of life concept to a theory. J Intellect Disabil Res. 2005; 49: 699-706.

21. Schalock RL, Keith KD, Hoffman K, Karan OC. Quality of life: its measurement and use. Ment Retard. 1989; 27(1): 25-31.

22. Salvador-Carulla L, Lucas R, Ayuso-Mateos JL, Miret M. Use of the terms "wellbeing" and "quality of life" in health sciences: a conceptual framework. Eur J Psychiat. 2014; 28(1): 55-70.

23. Wang M, Schalock RL, Verdugo MA, Jenaro C. Examining the factor structure and hierarchical nature of the quality of life construct. Am J Intellect Dev Disabil. 2010; 115(3): 218-33.

24. Emerson E, Hatton C. Self-reported well-being of women and men with intellectual disabilities in England. Am J Ment Retard. 2008; 113(2): 143-55. 
25. Stancliffe RJ, Lakin KC, Taub S, Chiri G, Byun SY. Satisfaction and sense of well being among Medicaid ICF/MR and HCBS recipients in six states. Intellect Dev Disabil. 2009; 47(2): 63-83.

26. Emerson E, Hatton C, Thompson T, Parmenter T. The international handbook of applied research in intellectual disability. Chichester, U.K.: Wiley. 2004.

27. Diener E, Emmons RA, Larson RJ, Griffin S. The satisfaction with life scale. J Pers Assess. 1985; 49: 71-5.

28. Verdugo MA, Schalock RL, Keith KD, Stancliffe RJ. Quality of life and its measurement: important principles and guidelines. J Intellect Disabil Res. 2005; 49(Pt 10): 707-17.

29. Schalock RL, Verdugo M, Jenaro C, Wang M, Wehmeyer M, Xu J, et al. A cross-cultural study of core quality of life domains and indicators: an exploratory analysis. Am J Ment Retard. 2005; 110: 298-311.

30. Lyons G. The Life Satisfaction Matrix: an instrument and procedure for assessing the subjective quality of life of individuals with profound multiple disabilities. J Intellect Disabil Res. 2005; 49(Pt 10): 766-9.

31. Gómez LE, Arias B, Verdugo MÁ, Tassé MJ, Brown I. Operationalisation of quality of life for adults with severe disabilities. J Intellect Disabil Res. 2015; 59(10): 925-41.

32. Cummins RA. Living with support in the community: predictors of satisfaction with life. Ment Retard Dev Disabil Res Rev. 2001; 7(2): 99-104.

33. Schalock RL, Brown I, Brown R, Cummins RA, Felce D, Matikka L, et al. Conceptualization, measurement, and application of quality of life for persons with intellectual disabilities: report of an international panel of experts. Ment Retard. 2002; 40(6): 457-70.

34. Voelker SL, Shore DL, Brown-More C, Hill LC, Miller LT, Perry J. Validity of self-report of adaptive behavior skills by adults with mental retardation. Ment Retard. 1990; 28(5): 305-9.

35. Schalock RL, Keith KD. Quality of life questionnaire manual. Worthington, OH: IDS publishing. 1993.

36. Mcvilly KR, Burton-Smith RM, Davidson JA. Concurrence between subject and proxy ratings of quality of life for people with and without intellectual disabilities. J Intellect Disabil Res. 2000; 25: 19-40.

37. Schmidt S, Power M, Green A, Lucas-Carrasco R, Eser E, Dragomirecka E, et al. Self and proxy rating of quality of life in adults with intellectual disabilities: results from the DISQOL study. Res Dev Disabil. 2010; 31(5): 1015-26.
38. Bertelli M, Bianco A, Rossi M, Scuticchio D, Brown I. Relationship between individual quality of life and family quality of life for people with intellectual disability living in Italy. J Intellect Disabil Res. 2011; 55(12): 1136-50.

39. Brown I, Renwick R, Raphael D. Quality of Life Instrument Package for adults with developmental disabilities. Toronto: Centre For Health Promotion, University of Toronto. 1997.

40. Hensel E, Rose J, Kroese BS, Banks-Smith J. Subjective judgements of quality of life: a comparison study between people with intellectual disability and those without disability. J Intellect Disabil Res. 2002; 46(Pt 2): 95-107.

41. Perry J, Felce D. Correlation between subjective and objective measures of outcome in staffed community housing. J Intellect Disabil Res. 2005; 49(Pt 4): 278-87.

42. Lachapelle Y, Wehmeyer ML, Haelewyck MC, Courbois Y, Keith KD, Schalock R, et al. The relationship between quality of life and self-determination: an international study. J Intellect Disabil Res. 2005; 49(Pt 10):740-4.

43. Balogh R, Ouellette-Kuntz H, Bourne L, Lunsky Y, Colantonio A. Organising health care services for persons with an intellectual disability. Cochrane Database Syst Rev. 2008; (4): CD007492.

44. Kendrick MJ. Getting a good life: the challenges for agency transformation so that they are more person centered. International journal of disability, community and rehabilitation. 2011. [online] 11 (1). Available from: http:// www.ijdcr.ca [accessed: 6 february 2015].

45. Dowling S, Manthorpe J, Cowley S. Working on person-centred planning: from amber to green light? J Intellect Disabil. 2007; 11(1): 65-82.

46. Bertelli M, Piva Merli M, Bianco A, Lassi S, La Malfa G, Placidi GF, et al. La batteria di strumenti per l'indagine della qualità di vita (BASIQ): validazione dell'adattamento italiano del Quality of Life Instrument Package (QoL-IP). Ital J Psychopathol. 2011; 7 (2): 205-12.

\footnotetext{
* Corresponding author:

Dr. Marco Bertelli

CREA-Research and Clinical Center-San Sebastian

Foundation, Misericordia di Firenze

Florence, Italy

Via del Sansovino, 176 - 50142 Firenze (Italy)

Tel. +390557392880

Fax +390557392879

E-mail: mbertelli@crea-sansebastiano.org

bertelli.fi@tiscali.it
} 